\title{
Relationship between a Doctor and a Patient with Mental Disorder
}

\author{
BLANKA KORES PLESNIČAR
}

\begin{abstract}
Quality relationship between a doctor and a patient with mental disorder is pivotal to successful treatment. The doctor, the patient and the environment contribute numerous factors to this relationship. There are also ethical and legal aspects to consider, and above all, it is intrinsically linked with the patient's trust. However, the relationship between a doctor and a patient with mental disorder may lead to crossing professional boundaries and even to their violation. Crossing boundaries means deviating from normal standards of practice that does not exploit or harm the patient, and can be even beneficial to the later; violation of boundaries is harmful to the patient and serves primarily for satisfying the needs of the doctor. Education and learning ethical norms and communication techniques represent the foundation of all professional relationships.

KEYWORDS: $\cdot$ doctor $\cdot$ patient $\cdot$ relationship $\cdot$ mental disorder $\bullet$ treatment
\end{abstract}

CoRresPondence AdDRess: Blanka Kores Plesničar, Ph.D., Professor, Senior Adviser, University Psychiatric Clinic Ljubljana, Studenec 48, 1000 Ljubljana, Slovenia, email: blanka.kores@psih-klinika.si. 
B. Kores Plesničar: Relationship between a Doctor and a Patient with Mental Disorder

The doctor who is most often in a relationship with a patient with mental disorder is undoubtedly a psychiatrist. Nowadays, both patient's and doctor's role are changing. This changing world of interpersonal relationships is perhaps easier explained from psychiatrist's point of view, as he is supposed to be professionally trained in the art of communication, but all new ethical, moral and legal aspects apply to all persons who are professionally involved in the treatment process and are entering into a relationship with a patient. Therefore, it is not always necessary to follow Cartesian paradigm on dualistic perspective of human being and division on mind and body on two separate entities; instead, it is necessary to consider and accept the man as a whole.

\section{Characteristics of modern relationship between a doctor and a patient with mental disorder}

Modern medicine is characterised by rapid technological progress. Relationship between a doctor and a patient is becoming increasingly technical and impersonal, as it is limited to a short patient's visit or hospitalization and is therefore often autocratic. A five- to fifteen-minute check-up, as permitted by modern standards, means that we can realistically see a patient with mental disorder every 6 months, and that we daily examine 20 to 30 patients. Consequently, we sometimes cannot even remember who the patient seated in front of us is, let alone any important events from his anamnesis, despite the fact that we are constantly emphasizing the importance of developments between the two persons who are entering into therapeutic relationship.

\section{How to achieve appropriate, good relationship between a doctor and a patient with mental disorder?}

A good relationship between a doctor and a patient with a mental disorder is the best protection against legal hassles, while such relationship is also the most acceptable from ethical point of view (Lakdawala, 2015).

Psychiatrists are also trained and educated in the field of communication and various types of interpersonal relationships, but numerous obstacles and factors may occur that prevent establishing of good connections between them and their patients (Lakdawala, 2015):

- Factors pertaining to the patient

- Factors pertaining to the treating physician

- Approach towards the patient

- Consents and priorities

- Malpractice

- Commercial aspects 


\section{a / Factors pertaining to the patient}

A patient comes to the doctor usually with certain symptoms, but also with expectations, their own beliefs, and sometimes also with false assumptions. Important factors are also the patient's life history, his ideas and methods of coping with illness. A patient with severe mental disorder generally does not show up voluntarily, but because of encouragement or pressure from his surroundings. He may come with expectations that the psychiatrist is a good listener, or a "mad scientist"; therefore, always with certain assumptions, if not with a kind of inverted stigma. He might present his symptoms in line with his personality structure, for example, histrionic or conversive persons emphasize their symptoms, persons with paranoid structure do not believe the doctor, and patients with schizophrenia are often convinced that they are not sick and that relatives, doctors or other persons are making up the illness. Even patient's relatives (especially of those with mental disorder) might approach doctors with hostility and mistrust, as their understanding of mental disorders is inadequate and their expectations are often exaggerated and unjustified (e.g. relatives of patients with dementia might be convinced that dementia was caused by hospitalization). On the other hand, some persons perceive the psychiatrist as authoritative saviour of all their problems, and are passively entering into the relationship, expecting that their problems will be solved by someone else. These relationships are even more complex in psychotherapy, as they are tied to a so-called transfer or specific psychotherapeutic relationship.

\section{b / Factors pertaining to the treating physician}

The same as with doctors in other specialities, education and training of psychiatrists is carried out in the framework of medical model. Despite the progress in many fields, psychiatry is still struggling to be recognized as equal medical science, and psychiatrists as "real doctors«. Since doctors do not significantly differ from general population in terms of personality and mental disorders, their personality traits (including illnesses) might have a significant impact on their relationships with patients, expectations of environment, their own social discontent and contra-transfer relationships in psychotherapy.

A bio-psycho-social model, which is already outdated and does not fulfil its purpose, is still prevalent in medicine and psychiatry. Psychological and social aspects are still present, but only barely (it is difficult to evaluate and influence them during the short-term visits to the clinic and with doctor's insufficient authorisations), and biological model, which is the easiest to implement, has prevailed. 


\section{c / Approach towards the patient}

The first rule is that the approach towards the patient is professional, without judgement, and open. It should not be restricted with prejudices, material gain, the importance of the patient or the patient's environment. The patient should be given enough time in a relaxed atmosphere, which is especially important for a patient with mental disorder - patients with depressive disorder need time to open up, while on the other hand, patients with schizophrenia tend to be suspicious, anxious, might also be aggressive and hostile towards their surroundings.

Many patients with mental disorder will not admit that they are mentally ill, because they do not recognize their symptoms as illness and are convinced that treatment is not necessary. They often retain this belief even after the end of treatment, since a symptom of lack of insight into illness often remains unchanged and cannot be cured. As a result, approximately $30 \%$ of patients with schizophrenia abandon their medications in 3 months after treatment, and $75 \%$ in the first year after treatment, all of which represents a beginning of a circular relationship between a patient and a doctor. Such relationship may also include a patient who is missing appointments, throwing away medication, accumulating or even selling drugs, denying his problems or emphasizing trivial problems. Doctors in such a difficult and frustrating relationship sometimes convince themselves that they are important or in control and that despite the "hopelessness", they are activating all their knowledge, expertise and energy for the patient. The primary task of a modern psychiatrist therefore is not prescribing drugs, but creation of new and more effective mechanisms of communication with the patient (and also with his relatives).

A technique of shared decisions recently developed in psychiatry, particularly in treatment of patients with schizophrenia. This decision-making system refers to participation of patients with schizophrenia in all medical issues, which should improve their compliance with treatment (Hamman et al., 2011). For efficiency and effectiveness of such relationship, it is essential that both the patient and the doctor strive towards the same goal. Communication in the course of treatment is highly dependent on doctor's knowledge and skills and on patient's familiarity with therapeutic options and procedures, his motivation to participate in treatment and on his perception that he is capable of participation in therapeutic decisions, i.e. his autonomy. The intention of this approach is to increase active participation of patients with severe mental disorders in treatment, to prevent blind compliance with doctor's instructions and to encourage patient's will to decide on his own behalf. The principle of shared decision is in psychiatry becoming a key element in transformation of mental-health system and an important factor of good clinical practice, irrespective of the fact that this principle cannot be implemented in all circumstances and with all patients - in case of lacking insight into illness or critical approach towards the illness, or if the patient is due to mental disorder endangering himself or others, even the most rational or empathetic approaches are ineffective. When all medical and legal conditions (Mental Health Act) are 
met, some other mechanisms are available to a psychiatrist in such cases, for example treatment in a secure ward without the patient's consent.

Protection of patient's personal data is extremely important for his relationship with the doctor, so "public discussion" on mental disorders of particular patients, including those in criminal or other legal procedures, should not be condoned.

Mental disorder often lasts a lifetime, so the patient and the doctor are also ageing together. Their relationship can only be beneficial, if it is relaxed, compassionate and equal. The doctor must step into the role of a responsible professional, and the patient must learn (at least in principle) that he is responsible for his own life and behaviour.

\section{d / Consents}

This area includes information on the illness, treatment, the patient's consent and competences, and area of interpersonal relationships, which has not yet reached its peak. In psychiatry, consents to treatment are well developed, since the consent to hospitalization and subsequent treatment is regulated by Mental Health Act, which requires extremely expedient doctor's assessment of patient's competence to voluntarily consent to treatment (only 4 hours for overall evaluation - unique in Europe!).This could sometimes be possible and sometimes not, as a 4-hour time frame for evaluation of mental disorder of a person entering treatment against his will is often unrealistic, professionally unacceptable or practically impossible (e.g. a drunk patient, who is loud, brawling, cursing and does not consent to hospitalization because of influence of alcohol, does not sober up in 4 hours - a motion is filed with the court for hospitalization against his will, but the patient wakes up in the morning, glad that he is warm, fed and safe, and, of course, agrees to hospitalization; a patient who is upset and scared because of hallucinations is not even aware of the importance of treatment in the first 4 hours, but calms down after 12 hours and agrees to hospitalization. Despite criticisms by the profession (in other countries this time frame spans from 24 to 76 hours), legislation, as well as "corrupt elements", remains unchanged.

\section{e / Commercial aspects}

Slovenia has a well-developed public health-care system, especially in the field of psychiatry, which is accessible 24/7 without a referral. On the other hand, there is a large supply of self-paid services in the field of psychotherapy, often with questionable "experts" and unsupervised ethics or other legally regulated policies. 
B. Kores Plesničar: Relationship between a Doctor and a Patient with Mental Disorder

Special circumstances in relationship between a doctor and a patient with mental disorder

\subsection{Crossings and violations of professional boundaries in relationship between a doctor and a patient}

With technological developments in medicine doctors are becoming less formal, but still respectful to patients, and are in relationship with a patient less frequently following rigid ethical rules. For this reason, they are more often crossing, and sometimes even violating, professional boundaries in relationship with a patient. Crossings of professional boundaries are departures from usual practice that are not exploitative or dangerous for the patient, or can even be helpful (e.g. compassionate touch), while violations of boundaries are harmful to the patient and mostly serve the doctor's, not the patient's needs (Galletly, 2004).

Professional boundaries represent a sharply defined edge of appropriate behaviour of a doctor, and serve two purposes (Marshall et al., 2008):

- They separate the therapeutic relationship from social, sexual, romantic, business, and other relationships

- They structure a relationship that distinguishes and maintains roles of the patient and the professional

American Psychiatric Association (APA) recognizes the fact that for psychiatry, a broader and more specific set of moral and ethical issues and dilemmas applies, compared to other medical fields; therefore, in addition to 9 standards that already apply for other areas, additional 39 standards were issued for psychiatry (American Psychiatric Association, 2008). The most important two are:

- A psychiatrist shall not gratify his or her own needs by exploiting the patient. The psychiatrist shall be ever vigilant about the impact that his or her conduct has upon the boundaries of the doctor-patient relationship, and thus upon the well-being of the patient. These requirements become particularly important because of the essentially private, highly personal, and sometimes intensely emotional nature of the relationship established between the patient with mental disorder and the psychiatrist.

- Because a patient with mental disorder tends to idolise and identify with his psychiatrist, the psychiatrist's conduct is especially important. The intensity of the relationship may activate sexual and other fantasies on the part of both the patient and the psychiatrist, while weakening the doctor's objectivity and control. Sexual activity with a current or former patient is unethical.

Research has shown that between 3 and $10 \%$ of doctors (predominantly male) had sexual relations with patients (Gartrell et al., 1986; Wilbers et al., 1992; Leggett, 1994). Sexually exploited patients are often reluctant to complain because of feelings of shame and guilt, fear that they will not be believed, or even concern 
over what will happen to the doctor if they complain about the exploitation. The problem is not unique to doctors, but also appears in other professional relationships, such as teacher - student, nurse - patient, supervisor - supervised and others.

Any abuse, not only sexual, has very negative effects on patients. Doctors are powerful authority figures who in abusing or exploiting a patient serve their own needs (in a similar way to a parent abusing a child). Abuse can lead to intense shame, guilt, depression, post-traumatic stress disorder, suicidal thoughts, drug abuse, break-up of their personal relationships, loss of employment, etc. Doctors who violate professional boundaries in a relationship in such manner often have their own mental problems or disorders, personality disorders (sociopath, narcissistic), are arrogant, under stress, are living with insufficient emotional support or in a difficult partnership. Such relationships most often involve male doctors and female patients. The most vulnerable are women who have previously been sexually abused. In one Australian research, it was found that two thirds of the 40 female patients who were abused in psychotherapy had a history of childhood abuse (Quadrio, 1996). Dependent and borderline personality disorders are also patient's risk factors.

A relaxed atmosphere in relationship with a doctor, for example, the use of first names, can lead to a relationship that is misinterpreted by the patients; the situation can get worse if a doctor becomes aware of the risk and wants to withdraw from such relationship. It is therefore necessary to determine in advance what is acceptable in relationship with the patient and what is not - one should be aware of his own weaknesses and shortcomings, should use objective documentation, build a satisfactory personal life, avoid dependence on relationships, not use patients for gratification of their own need of importance, etc.

A self-assessment questionnaire for doctors was developed in the United States to help them assess professional boundaries in relationship with a patient and identify possible crossings or even violations thereof (Galletly, 2004). It contains the following questions:

- Have you accepted for treatment individuals known to be referred by a current or former patient?

- Do you tell patients personal things about yourself in order to impress them?

- Do you take great pride in the fact that such an attractive, wealthy, powerful or important patient is seeking your help?

- Do you disclose sensational aspects of your patient's life to others (even when you are protecting the patient's identity)?

- Do you accept gifts or bequests from patients?

- Do you join in any activity with patients that may serve to deceive a third party (eg, insurance company)? 
- Do you ever use information learned from patients, such as business tips or political information, for your own financial or career gain?

- Do you make exceptions for your patients, such as providing special scheduling or reducing fees, because you find the patient attractive, appealing, or impressive?

- Do you make exceptions for your patient because you are afraid she/he will otherwise become extremely angry or self-destructive?

When a doctor faces problems with professional boundaries, he should seek help from colleagues or medical organizations. In Slovenia, this is impossible, partly because no suitable advisory body of Medical Chamber exists, and pressure by the media holds such power that any individual is publicly condemned and punished in advance. Foreign medical organizations have special committees that can respond in appropriate manner.

\subsection{Effect of coercive, protective and punitive measures on therapeutical relationship between a doctor and a patient with mental disorder}

The therapeutic relationship is a very important process-therapeutic factor in psychiatry and psychotherapy and plays a key role in patient's participation in treatment, adherence with therapeutic measures, resolution of problems and difficulties, medication adherence and the outcome of treatment. Quality therapeutic relationship has positive effects on patient's satisfaction, trust, and after all also to measures that prevent criminal behaviour.

Coercive, protective and punitive measures have negative effect on, or may even erode, therapeutic relationship, thereby preventing achievement of therapeutic goals (Höfer et al., 2015).Use of these measures may (e.g. in forensic psychiatry because of simultaneous implementation of judicial requirements) lead to a conflict in performing therapeutic role, since a doctor or a therapist in forensic psychiatry cannot focus exclusively on the patients' treatment (Höfer et al., 2015), but these relationships are not simple, neither are arguments, and there has been little research on this topic.

Höfer and collaborators (2015) have observed in their research that patients who are being voluntarily treated at a psychiatric hospital rated therapeutic relationship with their doctor or therapist significantly better than those treated in the same hospital against their will, while forensic psychiatric patients rated the quality of therapeutic relationship somewhere between the two groups. It seems likely that patients who were admitted involuntarily have harder time accepting certain restrictions than patients in forensic wards, who have been there longer and are better adapted. Therapeutic relationship is more significantly affected by symptoms of hostility and aggression in patients than by their legal status (such study could also be conducted in Slovenia). 
- Doctors often treat patients in technical manner, especially those with psychosis, dementia and substance abuse. We do not show them enough respect, we do not spend enough time with them, and we are listening less attentively. As every other patient, a patient with a mental disorder has his own peculiarities, which we must recognize and respond to them accordingly.

- Relationship between a doctor and a patient with mental disorder is versatile and subject to influence by many factors, for example family, environment, culture, media, legal provisions and ethics.

- A good therapeutic relationship between a doctor and a patient leads to a better outcome of the treatment, but we must keep in mind that a good therapeutic relationship might also be "too good".

\section{References}

American Psychiatric Association (2008) The principles of medical ethics with annotations especially applicable to psychiatry (Washington, DC: American Psychiatric Association).

Galletly, C. A. (2004) Crossing professional boundaries in medicine: the slippery slope to patient sexual exploitation, The Medical Journal of Australia, 181(7), pp. 380-383.

Hamann, J., Mendel, R., Meier, A., Asani, F., Pausch, E., Leucht, S., \& Kissling, W. (2011) "How to speak to your psychiatrist": shared decision-making training for inpatients with schizophrenia, Psychiatric Services, 62(10), pp. 1218-1221, doi: 10.1176/appi.ps.62.10.1218.

Höfer, F. X. E., Habermeyer, E., Mokros, A., Lau, S., \& Gairing, S. K. (2015) The impact of legal coercion on the therapeutic relationship in adult schizophrenia patients, PLoS One, 10(4), pp. e0124043, doi: 10.1371/journal.pone.0124043.

Lakdawala, P. D. (2015) Doctor-patient relationship in psychiatry, Mens Sana Monographs, 13(1), pp. 82-90, doi: 10.4103/0973-1229.153308.

Leggett, A. (1994) A survey of Australian psychiatrists' attitude and practices regarding physical contact with patients, Australia New Zealand Journal Psychiatry, 28(3), pp. 488-497.

Marshall, R. M., Teston, K., Myers, W. C. (2008) Psychiatrist/patient boundaries: When it's OK to stretch the line, Current Psychiatry, 7(8), pp. 53-62.

Quadrio, C. (1996) Sexual abuse in therapy: gender issue, Australia New Zealand Journal Psychiatry, 30(1), pp. 124-131.

Wilbers, D., Veenstra, G., van der Wiel, H. B. \& Weijmar Schultz, W. C. (1992) Sexual contact in the doctor-patient relationship in The Netherlands, British Medical Journal, 304(6841), pp. 1531-1534. 